\title{
Free vibration modelling of single-walled carbon nanotubes using the differential quadrature method
}

\author{
Belhadj A.*, Boukhalfa A., Belalia S.A. \\ Computational Mechanics Laboratory (MECACOMP), Department of Mechanical Engineering, \\ Faculty of Technology, University of Tlemcen, Algeria \\ Email: belhabdelkadir@gmail.com
}

\begin{abstract}
Carbon nanotubes (CNT's) has revolutionized the world of nanotechnology by their exceptional proprieties, which make them the core of many applications in several fields, many studies has been done to investigate their mechanical proprieties since their discovery. In this paper, the free vibration of a single walled carbon nanotube is studied in an elastic environment based on the theory of non-local elasticity and discretized by differential quadrature method (DQM); the effect of the surrounding medium on fundamental frequencies is discussed.
\end{abstract}

Keywords: Free Vibration, Carbon Nanotubes, Natural Frequency, Non-local Elasticity, Differential Quadrature Method, Euler-Bernoulli.

\section{INTRODUCTION}

The discovery of Carbon nanotubes (CNT's) in 1991 by Lijima [1], has enhanced the scientific research and opened new prospects for nanotechnology and materials science. Applications of Carbon Nanotubes are multiple including nano electro-mechanical systems (NEMS) [2], medical and biological devices, electronic chips, reinforced structures in menufacturing, singal precessing and measurment such as Atomic Force microscopy, this interest in CNTs is explained due to their exceptional mechanical [3], chemical, physical and thermal properties.

Considerable efforts has been devoted to invesitigate and understand the mechanical properties of CNT's using molecular dynamics [4] which need much time and high efficient computational resources, and Quantum mechanics by using the theory of non-local elsticity wich has been introduced by Eringen [5], this theory could investigate the dynamic behavior of nanoscale structures such as CNT's using the well-known beams models : Temoshenko beam and Euler-Bernoulli beam, Many studies have been done to understand the mechanical behaviour of CNT's[6,7,8,9, 10].

Various studies in the literature carried out the free vibration of single-walled carbon nanotubes embedded in elastic medium [11,12], SWCNT's with waviness [13], CNT's conveying fluids $[14,15,16]$.

Arda and Aydogdu [17] studied the effect of the surrounding environment on the torsional free vibration of carbon nanotubes, they showed that non-local parameter and the stiffness (viscol-elastic medium) effect on the non dimensioanl frequency in decreasing way, and the nanotube length effect the non dimensional frequency decresasingly and the non dimensional dumping increasingly. Bocko and Lengvarski [18] studied the bending vibration of armchair SWCNT under four types of boundary conditions using the theory of non-local elasticity; they showed the effect of nonlocal parameter on the bending frequencies of the CNT.

In this paper, the equation of motion is disceritized using the differential quadrature method (DQM) which was introduced for the first time in 1972 by Bellman et al. [19], since that, it has been widely used for solving multiscale structures dynamic, Malik and bert [19] had employed it for solving different problems in applied mechanics.

\section{MATHEMATICAL MODELLING}

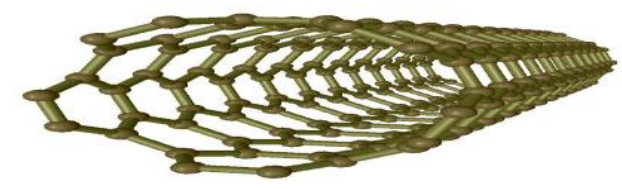

Figure 1. Molecular diagram of a SWCNT.

The present paper investigates the transverse free vibration of a single-walled carbon nanotube (SWCNT) embedded in an elastic medium described as a Winkler-type elastic foundation. The elastic medium is assumed to act as a pressure acting on the outer layer of the SWCT.

The figure. 1 illustrates the molecular diagram of a SWCNT modelled by a molecular dynamic software.

Based on Euler-Bernoulli beam theory, the displacement field at any point can be expressed as: 
$u_{x}(x, t)=u(x, t)-z \frac{\partial w(x, t)}{\partial x}$

$u_{z}(x, t)=w(x, t)$

The linear strain-displacement relations for the EulerBernoulli beam are expressed as:

$\varepsilon_{x x}=\frac{\partial u}{\partial x}-z \frac{\partial^{2} w}{\partial x^{2}}$

$\varepsilon_{z z}=\varepsilon_{x z}=0$

The law of Hook expressing the strain as following:

$\sigma_{x x}=E_{x} \varepsilon_{x x}=E\left(\frac{\partial u}{\partial x}-z \frac{\partial^{2} w}{\partial x^{2}}\right)$

We define herein the kinetic and strain energies, respectively:

$K e=\frac{1}{2} \iint_{0}^{L S} \rho\left(\delta \dot{u}^{2}+\delta w^{2}\right) d S d x$

$S e=\int_{0}^{L S}\left(\int_{x x} \delta \varepsilon_{x x}\right) d S d x$

Then, the governing equations are obtained by deriving the above energies expressions based on the Hamilton's principle defined as follwing:

$\int_{T 1}^{T 2}[K e+S e]=0$

Two equation of motion are obtained: longitudinal and transverse inertia, only transverse vibration equation containig the deflection $\mathrm{w}(\mathrm{x}, \mathrm{t})$ is the aim of this study, expressed as :

$\rho S \frac{\partial^{2} w}{\partial t^{2}}+E I \frac{\partial^{4} w}{\partial x^{4}}=0$

By introducing the surrounding elastic medium law:

$\rho S \frac{\partial^{2} w}{\partial t^{2}}+E I \frac{\partial^{4} w}{\partial x^{4}}=F(x)$

$\mathrm{F}(\mathrm{x})=-\mathrm{kw}$

Due to nearest neighbor interaction and beyond the single lattice in the sense of lattice average stress and strain. Eringen [5] improved that the stress of a reference point of the body depends on the strain of other adjacent points of this body. The non-local elasticity is expressed as:

$$
\left[1-(e, a)^{2} \nabla^{2}\right] \sigma^{n l}=\sigma^{l}
$$

By applying the constituve theory of Eringen, we obtain: $\rho S\left[\frac{\partial^{2} w}{\partial t^{2}}-\left(e_{0} a\right)^{2} \nabla^{2} \frac{\partial^{2} w}{\partial t^{2}}\right]+E I \frac{\partial^{4} w}{\partial x^{4}}+K[w$

$\left.-\left(e_{0} a\right)^{2} \nabla^{2} w\right]=0$

The transverse deflection law is defined as:

$w(x, t)=W \cdot e^{i \omega t}$

We define the follwing demonsionless paramaters:

$\Omega^{2}=\frac{\rho S \omega^{2} L^{4}}{E I}, \bar{K}=\frac{k L^{4}}{E I}, \mu=\frac{e_{0} a}{L}, \xi=\frac{x}{L}$

The dimensionless equation of motion is transformed to:

$\bar{K}\left(1-\mu^{2} \frac{d^{2}}{d \xi^{2}}\right) W-\Omega^{2}\left(1-\mu^{2} \frac{d^{2}}{d \xi^{2}}\right) W+\frac{\partial^{4} w}{\partial \xi^{4}}=0$

The resolution of the above equation consists in solving the follwing eigen-problem:

$\left(G-\Omega^{2} M\right) q=0$

The frequency of the Euler-Bernoulli beam eigen-problem is:

$\Omega=\sqrt{\frac{G}{M}}$

\section{DIFFERENTIAL QUADRATURE METHOD}

The discretization of the governing equation is done using the semi-analytical procedure: differential quadrature method (DQM), It transforms the differential equation to a set of equivalent simultaneous equations using the weighting coefficients summation.

If $\frac{\partial W}{\partial \xi}=\sum_{j=1}^{W} A_{i_{j} j}:$ is the first derivative, we define it as:

$A_{i_{i} j}=\frac{P\left(\xi_{i}\right)}{\left(\xi_{i}-\xi_{j}\right) \cdot P\left(\xi_{j}\right)} i_{i j}=1,2 \ldots N_{s} i \neq j$

with:

$P\left(\xi_{i}\right)=\prod_{j=1}^{N}\left(\xi_{i}-\xi_{j}\right) \quad i \neq j$

and when

$i=j \quad A_{\mathrm{i}_{j} j}=A_{\mathrm{i}_{, j}}=\sum_{j=1}^{N} A_{\mathrm{i}_{i}, k} \quad i=1,2 \ldots N_{s} i \neq k, i=j$

Similarly, second-, third- and fourth-order partial derivative are expressed as

$\sum_{i_{j} j=1}^{W} B_{i_{j} j}=\sum_{k=1}^{W} A_{i k} \cdot \sum_{k=1}^{W} A_{k_{i} j}:$ is the second derivative;

$\sum_{i_{j} j=1}^{N} C_{i, j}=\sum_{k=1}^{N} B_{i k} \cdot \sum_{k=1}^{N} A_{k j j}:$ is the third derivative;

$\sum_{i_{j} j=1}^{N} D_{i_{j} j}=\sum_{k=1}^{N} B_{i k} \cdot \sum_{k=1}^{N} B_{k, j}:$ is the fourth derivative.

The discretized governing equation is defined as: 
$\bar{K}\left(1-\mu^{2} \sum_{j=1}^{W} B_{i, j}\right) W_{j}-\Omega^{2}\left(1-\mu^{2} \sum_{j=1}^{W} B_{i, j}\right) W_{j}$

$+\sum_{j=1}^{W} D_{i, j} W_{j}=0$

The resolution of the problem using DQM is done at a set of points satisfying the Chebyshev-Lobatto-Gauss, which satisfies:

$\xi_{\mathrm{i}}=\frac{1}{2}\left(1-\cos \frac{(i-1) \pi}{N-1}\right) i=1,2 \ldots N$

Various boundary conditions are implemented to investigate the transverse free vibration of a SWCNT embedded in an elastic medium; we define the following boundary conditions:

clamped-clamped beam (C-C)

$w(x=0)=\frac{\partial w(x=0)}{\partial x}=w(x=L)=\frac{\partial w(x=L)}{\partial x}$

clamped-pinned beam (C-P)

$w(x=0)=\frac{\partial w(x=0)}{\partial x}=w(x=L)=\frac{\partial^{2} w(x=L)}{\partial x^{2}}$

pinned-pinned beam (P-P)

$w(x=0)=\frac{\partial^{2} w(x=0)}{\partial x^{2}}=w(x=L)=\frac{\partial^{2} w(x=L)}{\partial x^{2}}$

\section{NUMERICAL RESULTS AND DISCUSSION}

A Matlab code has been developed to compute fundamental frequencies of the SWCNT, boundary conditions are defined by setting the corresponding weighting coefficients to zero at the ends $(\xi=0)$ and $(\xi=1)$. The SWCNT proprieties are taken as following:

$m=3.25 \times 10^{-24} \mathrm{~kg} ; \mathrm{d}=1.356 \mathrm{~mm} ; E I=358.66 \mathrm{~kg} \cdot \mathrm{mm}^{\mathrm{a}} / \mathrm{s}^{2}$

The dependency of natural frequencies on the length of the SWCNT is illustrated in Figure.2 for the clamped-pinned beam; it is clearly remarkable that the first four frequencies converge to a constant value at the end of the SWCNT.

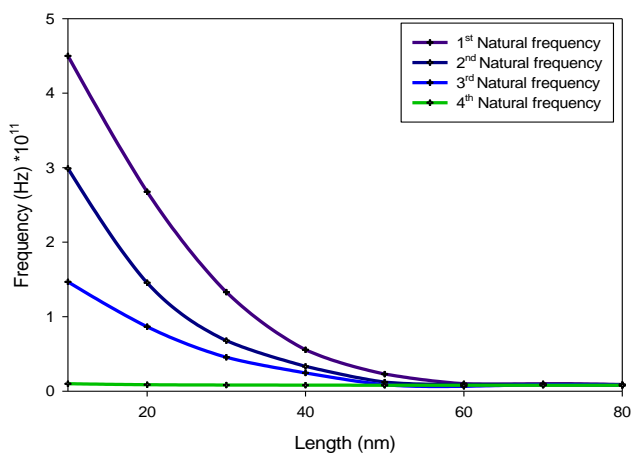

Figure 2. First four natural frequencies of Clamped-pinned beam for $\mathrm{K}=0$

In following, the effect of non-local parameter and the elastic medium (Winkler-type) is studied, Figure. 3 shows that the frequency parameter of clamped-clamped beam at a null elastic parameter decreases from the local value $(\sim 21.4)$ to a stable value corresponding to $\mu=0.03$. The effect of the nonlocal parameter is clearly remarkable on the frequency parameters corresponding to $\mathrm{K}=200$ where the difference of frequency parameter is 0.5 when $\mu$ ranging from 0.01 to 0.04 .

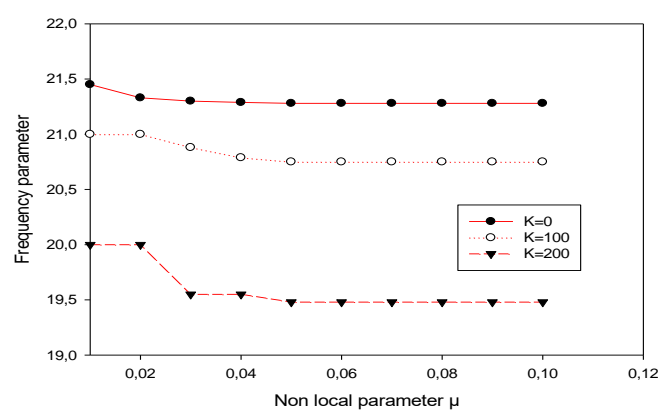

Figure 3. Frquency parameters versus non-local parameter of C-C beam

For the clamped-pinned beam in Figure.4, all frequency parameters with different elastic values decrease when $\mu$ ranging from 0.01 to 0.03 . We can conclude that the frequency parameters are independent of the non-local parameters when $\mu \geq 0.03$.

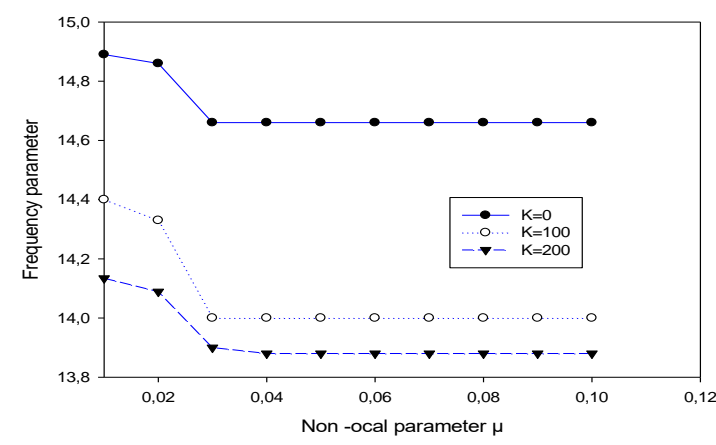

Figure 4. Frquency parameters versus non-local parameter of C-P beam

The frequency parameters decrease with the increasing of the elastic medium parameter K. All remarks for the Figure.4 remain valid for the Figure. 5 which illustrate the variation of frequency parameters with the non-local parameter and the elastic medium parameter for a pinned-pinned beam.

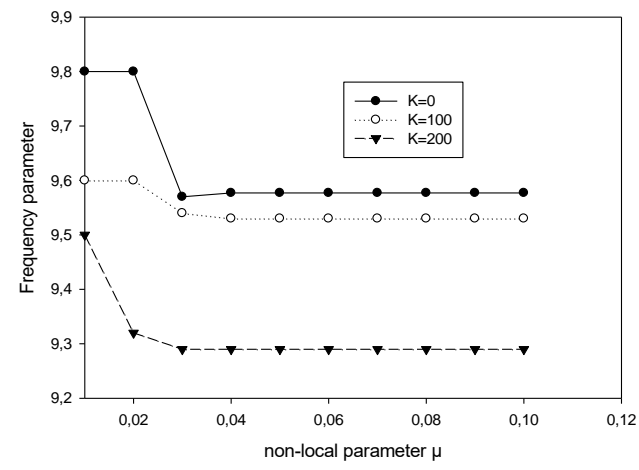

Figure 5. Frquency parameters versus non-local parameter of P-P beam 


\section{CONCLUSIONS}

Free transverse vibration of a single-walled carbon nanotube is investigated in detail, based on the non-local theory of elasticity by using the differential quadrature method. The non-local Euler-Bernoulli beam model is taken in consideration to study the vibrational behavior of the SWCNT under different boundary conditions, the nanotube length affected fundamental frequencies of SWCNT decreasingly; considered values of non-local parameter show a stability of frequency parameters less small than the local parameters. By increasing the elastic medium, the frequency parameters decrease.

\section{REFERENCES}

[1] Iijima S. (1991). Helical microtubules of graphitic carbon, Nature, Vol. 345, pp. 56-58. DOI: $\underline{10.1038 / 354056 \mathrm{a} 0}$

[2] Cha S.N., Jang J.E., Choi Y., Amaratunga G.A.J., et al. (2005). Fabrication of a nanoelectromechanical switch using a suspended carbon nanotube, Applied Physics Letters, Vol. 86, 083105. DOI: 10.1063/1.1868064

[3] Lu J.P. (1997). Elastic properties of carbon nanotubes and nanoropes, Physical Review Letters, Vol. 79, pp. 1297-1300. DOI: 10.1103/PhysRevLett.79.1297

[4] Chong K.P. (2008). Nano science and engineering in solid mechanics, Acta Mechanica. Solida Sinica, Vol. 21, No. 2, pp 95-103. DOI: 10.1007/s10338-008$0812-7$

[5] Eringen A.C. (1972). Nonlocal polar elastic continua, International Journal of Engineering Science, Vol. 10, No. 1, pp. 1-16. DOI: 10.1016/0020-7225(72)900705

[6] Murmu T., Pradhan S.C. (2009). Buck ling analysis of a single-walled carbon nanotube embedded in an elastic medium based on nonlocal elasticity and Timoshenko beam theory and using DQM, Physica $E$ 2, Vol. 41, No. 7, pp. 1232-1239. DOI: https://doi.org/10.1016/j.physe.2009.02.004

[7] Heireche H., Tounsi A., Benzair A., Maachou M., Adda Bedia E.A. (2008a). Sound wave propagation insingle-walled carbon nanotubes using nonlocal elasticity, Physica E, Vol. 40, pp. 2791-2799. DOI: 10.1016/j.physe.2007.12.021

[8] Semmah A., Tounsi A., Zidour M., Heireche H., Naceri M. (2014). Effect of chirality on critical buckling temperature of a zigzag single-walled carbon nanotubes using nonlocal continuum theory, Fullerenes, Nanotubes. And Carbon Nanostructures, Vol. 23, pp. 518-522. DOI: 10.1080/1536383X.2012.749457

[9] Houmat A. (2015). Nonlinear free vibration of nonprismatic single-walled carbon nanotubes by a nonlocal shear deformable beam p-element, Acta Mechanicca, Vol. 2271, No. 4, pp. 1051-1065. DOI: 10.1007/s00707-015-1507-z

[10] Belhadj A., Boukhalfa A., Belalia S.A. (2017). Carbon nanotube structure vibration based on non-local elasticity, Journal of Modern Materials, Vol. 3, No. 1, pp. 9-13. DOI: $10.21467 / \mathrm{jmm} .3 .1 .9-13$

[11] Gupta S.S., Bosco F.G., Batra R.C. (2010). Wall thickness and elastic moduli of single-walled carbon nanotubes from frequencies of axial, torsional and

inextensional modes of vibration, Computational Materials Science, Vol. 47, No. 4, pp. 1049-1059. DOI: $10.1016 /$ j.commatsci.2009.12.007

[12] Pirmohammadi A.A. et al. (2014). Modeling and active vibration suppression of a single-walled carbon nanotube subjected to a moving harmonic load based on a nonlocal elasticity theory, Applied Physics A, Vol. 117 , No. 3, pp. 1547- 1555. DOI: $10.1007 / \mathrm{s} 00339-$ $\underline{014-8592-\mathrm{Z}}$

[13] Soltani P., Kassaei A., Taherian M.M. et al. ( 2012). Vibration of wavy single-walled carbon nanotubes based on nonlocal Euler Bernoulli and Timoshenko models, International Journal of Advanced Structural Engineering, Vol. 4, No. 3, pp. 1-10. DOI: 10.1186/2008-6695-4-3

[14] Lee H., Chang W. (2009). Vibration analysis of a viscous-fluid-conveying single-walled carbon nanotube embedded in an elastic medium, Physica. E, Vol. 41, pp. 529-532. DOI: 10.1016/j.physe.2008.10.002

[15] Lee H., Chang W. (2009). Vibration analysis of a viscous-fluid-conveying single-walled carbon nanotube embedded in an elastic medium, Physica E, Vol. 41, pp. 529-532. DOI: 10.1016/j.physe.2008.10.002

[16] Wang L., Ni Q. (2009). A reappraisal of the computational modelling of carbon nanotubes conveying viscous fluid, Mechanics Research Communications, Vol. 36, pp. 833-837. DOI: 10.1016/j.mechrescom.2009.05.003

[17] Arda M., Aydogdu M. (2015). Analysis of free torsional vibration in carbon nanotubes embedded in a viscoelastic medium, Advances in Science and Technology Research Journal, Vol. 9, No. 26, pp. 283. DOI: $10.12913 / 22998624 / 2361$

[18] Bock J.O., Lengvarský P. (2014). Vibration of singlewalled carbon nanotubes by using nonlocal theory, American Journal of Mechanical Engineering, Vol. 2, No. 7, pp. 195-198. DOI: 10.12691/ajme-2-7-5

[19] Bellman R., Kashef B.G., Casti J. (1972). Differential quadrature: a technique for the rapid solution of nonlinear partial differential equations, Journal of Computational Physics, Vol. 10, pp. 40-52. DOI: $\frac{10.1016 / 0021-9991(72) 90089-7}{}$

[20] Bert C.W., Malik M. (1996). Differential quadrature method in computational mechanics: a review, Applied Mechanics Reviews, Vol. 49, pp. 1-27. DOI: $\underline{10.1115 / 1.3101882}$

\section{NOMENCLATURE}

A

a

$\mathrm{B}$

C

$\mathrm{D}$

d

$\mathrm{E}$

$\mathrm{e}_{0}$

G

$\mathrm{K}$

$\bar{K}$

$\mathrm{Ke}$
First derivative

Internal caracteristic length

Seconde derivative

Third derivative

Fourth derivative

Nanotube diameter (nm)

Young's modulus (PA)

Constant depends on the material Stifness matrix

spring constant relative to the elastic medium (Winkler type) (N/m2)

Non dimensional spring constant

Kinetic energy $(\mathrm{J})$ 
Nanotube length (nm)

Carbon nanotube mass $(\mathrm{kg})$

Mass matrix

Sampling points

Displacement vector

Polynomials of weighting coefficients

Section (m2)

Strain energy $(\mathrm{J})$

Time (s)

displacement (nm)

Transverse amplitude

Transverse deflection

Axial component corresponding to axial axis

Transverse component

\section{Greek symbols}

$\sigma$

$\rho$

$\partial$

$\xi$

$\Omega$

$\mu$

$\omega$

\section{Subscripts}

i

$\mathrm{j}$

$\mathrm{k}$

1

$\mathrm{nl}$
Strain (N/m2)

Density $(\mathrm{kg} / \mathrm{m} 3)$

Partail derivative

Dimensionless axial component

Dimensionless parameter corresponding to Frequecy

Non-local parameter

Natural frequency $(\mathrm{Hz})$

Longitudinal matrix component Transverse matrix component Intermediar matrix component Local

Non-local 